\title{
Scope specification of coccidiosis in the poultry on researchers
}

\begin{abstract}
The poultry is important in Socio economy of the worlds. Poultry gave us many valuable products such meat and egg that important in nutritional value and health status. The poultry defined small scale keeping for many rural and livelihood in their household income. They prefer to keep in different condition such as free range extensive, backyard, semi intensive and intensive in their chickens because the poultry provide the household income for the sale of live birds and eggs. According to consumption of poultry provide valuable source of protein in the diet. The farmer prefers for raising poultry in their rapid growth and their daily product. As many diseases challenged in the production and productivity as well the health status of poultry like other livestock disease. Also, there is gastrointestinal parasites that endemic in many countries of the world. These parasitic diseases of poultry include coccidiosis. The coccidia Eimeria. This Eimeria live and replicate intestinal mucosa then caused damage. This Eimeria cause destructive absorption of the intestine leading dehydration and blood loss, and cause immune suppression and cause infection with other opportunistic bacterial infections. So, this review deals to highlight and specify information about the Coccidiosis in poultry for their life cycle, morphology, epidemiology, risk factor, clinical sign and pathology, postmortem lesion, diagnosis and treatment and control in the coccidiosis was briefly discussed, Further study of molecular and easy diagnostic test of Eimeria and framework of strategic control and eradication of this disease from the world.
\end{abstract}

Volume 5 Issue 2 - 2020

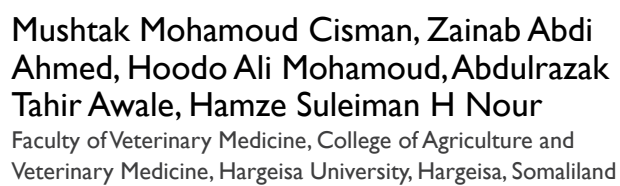

Correspondence: Hamze Suleiman H Nour, Faculty of Veterinary Medicine, College of Agriculture and Veterinary Medicine, Hargeisa University, Hargeisa, College of Veterinary Science, Department Tropical Veterinary Medicine, Mekelle University, Mekelle Ethiopia. Ministry of Livestock and Fishier Development, Hargeisa Somaliland, Tel +252634756262. Email Hamse05@gmail.com

Received: October 14, 2020 | Published: December 14, 2020

Keywords: scope, specification, poultry, coccidiosis, researcher, review

\section{General description on poultry coccidiosis}

The protozoan parasites that caused many challenged and disease in poultry include Coccidiosis. The parasite replicates and multiply intestinal tract after multiplication and parasitic load that was causes tissue damage. The tissue damaged and interfere with activity of the intestinal such as food digestion and its nutrition absorption, and causing blood loss this leads and causes dehydration. There a lot of bacteria that was life the intestinal of chicken, after tissue of intestinal damage there is emerging bacterial opportunistic that cause infection; these bacterial include Clostridium and Salmonella. Coccidiosis parasite develop in the chickens immune system with immune suppression and produce more severe problems. Coccidiosis cause interfere and exacerbate with infections of Mark's disease and infectious Bursal disease with their development of the immune of the birds. ${ }^{1}$

Eimeria species is causative agent of poultry coccidiosis and is member by a protozoan parasite and is known poultry disease that related in chicken. This species has effect part of intestinal tract of poultry recorded from the world. ${ }^{2}$

There is cross immunity between of species that produce effective immunity of the chicken against in each species of coccidiosis and their host specific and able to produce. The species include Eimeria was most pathogenic and economic loss. Species of Eimeria tenella is importance in gastrointestinal and its host location was caecal and causes loss of blood that's called bloody type of Coccidiosis. The other species $E$. necatrix also causes intestinal damage and causes blood coccidiosis. Consequently E. acervuline and E. maxima was intestinal parasite in long time or chronic form of coccidiosis. ${ }^{3}$

\section{Morphology}

Ovoid shape of their oocytes has the Eimeria eggs, egg size $(30.5 \times 20.7 \mu \mathrm{m})$ this largest egg in Eimeria maxima, the second was Eimeria mivati $(15.6 \times 13.4 \mu \mathrm{m})$ and the smallest egg of Eimeria species was Eimeria mitis $(15.6 \times 14.2 \mu \mathrm{m})$ Eimeria species E. tennela, E. maxima, E. acervulina, E. hagani and E. burnetti are ovoid while E. necatrixis oblong, their oocyst size, shape, color are helpful identification of Eimeria species. ${ }^{4}$ Further species identification includes microscopic lesions of localized intestine parasitized, minimum sporulation time, minimum prepatent period, schizonts size and area in which develops, with their parasite in the epithelial cell their protective immunity trails against coccidia infections. ${ }^{4}$

\section{Life cycle}

The sexual development in all Eimeria species has two or more generation of an asexual development known as schizogony, the live cycle of all Eimeria involves two or more generation of an as sexual development followed by a sexual phase this formed by gametogonium this results in formation of oocyte of different species of Eimeria. ${ }^{5}$

The Eimeria continuous their growth and development, after production of oocyte into intestine the affective stage, sporulated oocyst, is ingested and localize the gut, they make action of mechanical and chemical factors such as (bile salt and trypsin). This effective stage release of sporocysts then sporozoites in duodenal lumen and localized. This effective of sporozoites reaches in whole length of the alimentary tract and invade mucosa tissue, after production of sporozoites followed second phase of intracellular growth and asexual 
multiplication with periodic release of merozoites entering in to the sexual phase of the life cycle of Eimeria species this process called as gametogenesis. ${ }^{6}$

Also, during growth and development of the Eimeria invades cells and development their sexual growth in two phases either macro-micro gametes. This rise into a single macrogamete whereas the male gametocyte matures and ruptures, large number of minute biflagellate micro-gametes (Figure 1). This process formed into micro gametes. This micro gamete has thickened wall forms around the macrogametes, this forming a zygote the macro gametes is fertilized by microgametes. This is immature oocyst or young stage. ${ }^{7}$

The life cycle is important feature with quite rapid occurred with 4-5 days about their prepatent period of life cycle (which varies slightly with species) and involves colossal multiplication. Thousands or even millions of oocytes formed from one ingested oocyte though there was variation degree of oocytes of species. ${ }^{8,9}$

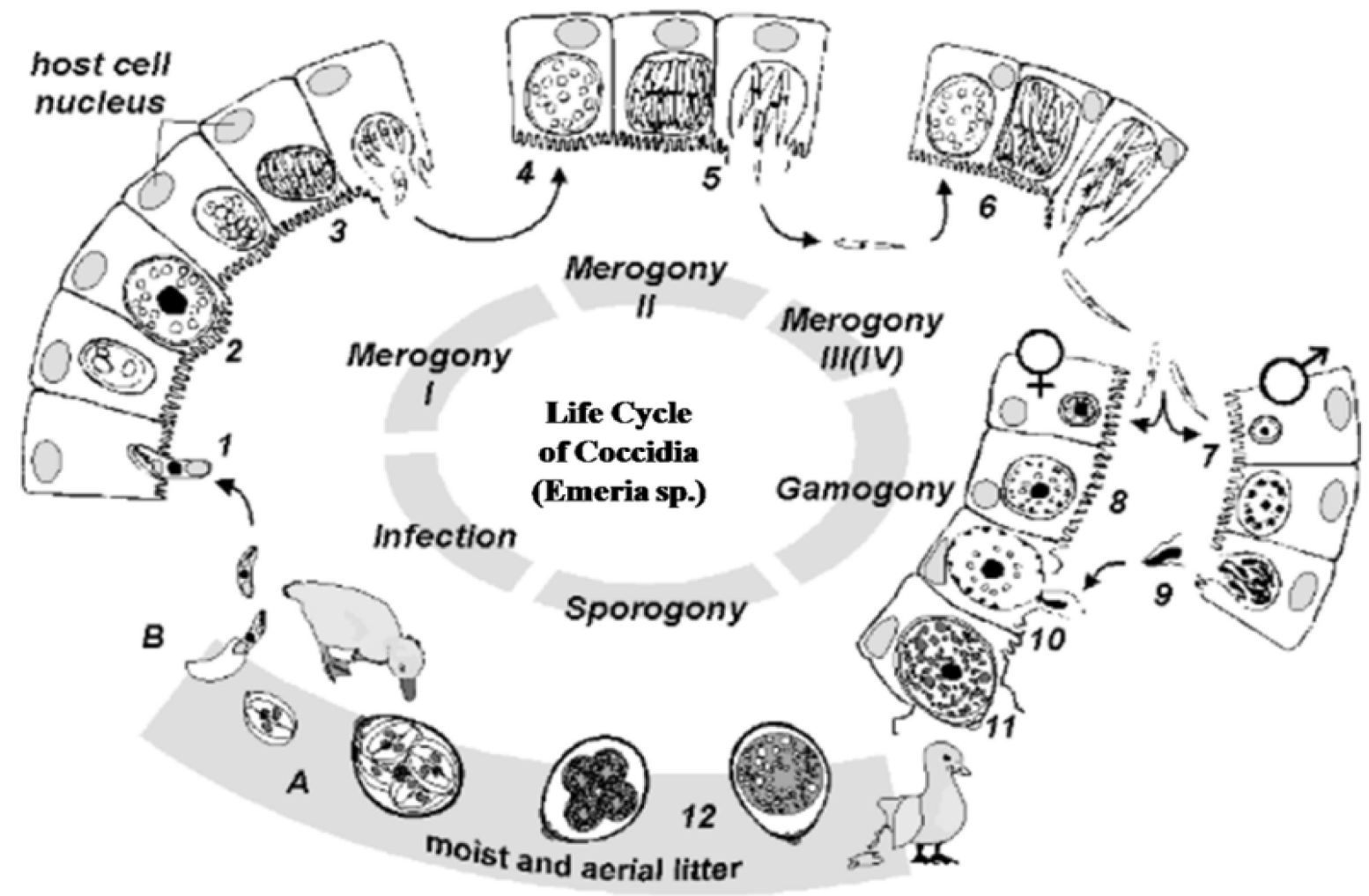

Figure I This tells you there is variation of stages of Eimeria are different in and outside intestinal cells [Source: 38 ].

Eimeria of species their control of biochemical and genetic mechanisms development cell not clear or unknown. There was drug resistance of $E$. tenella, ${ }^{10}$ previous studies show that there were two linkage groups related their genetical or molecular studies have been identified and the result mapped was parasite chromosomes 1 and Chromosomes 2. This result identified other genetic loci related for regulation of life cycle of E. tenella for further studies abut researchers, ${ }^{11}$ also, recently identified a gene (ets3a) whose developmentally expression is regulated which controlled by the life cycle and sexual development of E. tenella. ${ }^{11}$

\section{Epidemiology}

The risk factors contribute prevalence of coccidiosis and their geographical distribution include high animal density cramped in area size such as on a small space, the temperature, high humidity, and age was risk factors contribute the prevalence of coccidiosis and there is variation of poultry among the age groups, feed inconstant, quantity and quality of feed as well amount of feed and other risk factors include resistance to disease in poultry species and general health condition of chickens. When the disease attached the chickens or bird at first time the immune response is strong and fight against disease or infection this process completed within 10 days depend on the degree of infection and status of their overall health status of chicken to develop and engulf the infection agent for proper mechanism of immune response of chickens or birds. ${ }^{12}$

The coccidia always lives in free environment and was abundant under farming condition for coccidiosis. ${ }^{9}$ The oocytes found from food and water contaminated by feces of infected animal and chickens for several days or weeks and even months that oocyte shed by the feces, under proper condition oocytes hatched and populated within 2 days and develop into infective stages. Under extensive grazing chickens or farming chicken they make feeding the pick-up with feed or other materials on the ground and litter that was used for bedding in the houses with oocytes that ingested and shed by the feces. ${ }^{8}$ Normally the coccidia found in contaminated facility and equipment, vehicle and shed objective that comes from poultry or bird in coccidiosis passed with feces and hatched after found favorable condition.?

The house of poultry inside and outside of the farm there found vermin by invertebrates with their assist for mechanical ventilation 
systems serve to scatter oocytes outside build and distributed oocytes where they can find suitable condition to develop an effective stage. As we mentioned above vehicle, personal contribute distribution of oocytes of coccidiosis to other farms. ${ }^{13}$ Other vectors contribute the distribution of coccidian oocytes is important by the peoples, ${ }^{14}$ other factors contribute oocyte dissemination could be manure clinging to shoes, utensils from one pen or farm to the others. Lastly, the factors contribute dissemination of oocytes within the farms include beetles, flies, cockroaches, pets, rodents and mild birds have mechanical role for incriminated source of spread of coccidian oocytes. ${ }^{4}$

\section{Risk factors contribute occurrence of coccidiosis in poultry}

The different species of Eimeria and their oocytes its size and dose of infection has significance emergence and occurrence of coccidiosis in poultry as well their short of prepatent period of the Eimeria species and high biotic potentials increase oocytes in the litter rises rapidly. ${ }^{9}$ During replication process of coccidian oocytes has capacity rapid replication within the host with subsequent high level of the parasites within the susceptible host and high environment contamination of occurrence of coccidiosis. ${ }^{15}$ Eimeria oocytes have sporulated coccidian oocytes to harsh environmental condition have capacity to reproduce viability outside host, weak immunity and lack of cross immunity between Eimeria species is predisposing factor for chicken or bird to enhance infection and rapid occurrence disease outbreaks causes different host species and different Eimeria species. ${ }^{16,17}$

\section{Host related risk factors}

According the age of chicken or birds, coccidia always infected about 3-4 weeks of ages, frequently, the coccidiosis was disease of young and is more susceptible when there was exposed before, and can be infected at any time, if they previously occur the disease. But their dangerous state is taken over times, ${ }^{8}$ the space and pens increase density animal cramped on a small space, age of the bird at the time of first infection and the number of passages of infection as well the immune response against disease will develop when infection get the body of the birds and lastly week the immune of bird and their ability. $^{12}$

\section{Pathology and clinical finding}

The infectious forms of the causative agent are oocytes in the form of spores. Birds get infection through contamination of feed and water, also, ingestion oral route of the birds. Infection forms after ingested of oocytes of sex cyst, Sporozoan gets infected epithelial cells of intestine of the birds. The movement and transferred with help of intraepithelial lymphocytes to reach primary location of Eimeria species to make lesion damage after colonization of degree of infections. ${ }^{18,19}$

The dose of infection of successful replication of coccidian oocytes depends pathogenicity of coccidia within the host in the parasites. Schizogonic phase is start process of pathogenic process of parasites development, their first generation schizonts and is negligible in the pathogenic process. During second generation of schizonts is important effective stage of pathological condition. The inflammation, mucus desquamation, capillary rupture and hemorrhage was the result of pathogenic within deep cells depend on the development of the oocytes. The companied with several clinical symptoms of this disease its pathological effect of the disease leads dangerous outcome could be death of chickens or birds. Other symptoms include toxemia, hemorrhage loss of blood about $(60,80$ percent of the blood volume the consequence of gangrene or rupture of the walls of the intestinal. ${ }^{20}$

Genetical host variation, nutritional factors, concurrent disease, and strain of coccidian. influenced pathogenesis occurrence of coccidiosis. The pathogenic of coccidiosis located lamina propria and crypts of epithelium of the small intestine and ceca and E. necatrix and E. tenella respective in the host specific location then lead cause damage in these tissue with extensive hemorrhage. Epithelial cells lining preferred in lining the villi, ${ }^{20}$ coccidiosis is a disease with gut and damage the gut that allows occur secondary bacterial infection. ${ }^{8}$ The gametogony, and development replication of oocytes, last generation of merogony released mature merozoites with mucosal surface that associated with tissue destruction of clinical signs. The severe infection of mucosal epithelium is sloughed off and stop absorption and interfere digestion process. . $^{16,17,21}$

This causes yellow diarrhea was first and most frequent symptoms. As the disease increase progresses, causes blood loss in feces, their appearance of feces was red or resemble the color of chocolate. Bloody deposits with covered around feathers and cloacae. Blood stained with feces. Infection progress the birds survive first few days up to 10 to 15 days during that time. During time that birds immune fighting infection, the birds rapidly loss weigh and thirsty the birds, thus clinical manifestation of the disease began when second generation of schizonts start rapidly replicate, growth mature and release the second generation of merozoites. ${ }^{21}$

\section{Necropsy findings}

After damage of tissue of gut become thickening walls of intestine which make them feel like sausage. Their surface of tissue of the gut hemorrhages and found streaks and their light color spot on surface tissue of intestine. ${ }^{8}$ The Eimeria species have different mechanism which causes lesion and different type, location of the tissue of intestine. For instance, the E. acervuline affected the upper parts of the small intestine, seems small red spot and white bands that found in the small intestine. Secondly, the E. maxima affect the whole entire small intestine, this damage causes watery finally, have blood and mucus. The E. tenella affect blind sacks of the gut, the intestine look thickened and ballooned with red pinpoint lesion of the gut, then intestine filled with blood and pus then turned into a solid core 8 . The damage of tissue and walls of the gut become thickened indicating retention of fluid (edema). Damage blood vessels in the lumen of the intestine indicated blood loss or hemorrhage and merely retention of an excessive amount of blood in the tissue (hyperemia), the development of immune response also, infiltration with various body reaction. ${ }^{5}$

\section{Diagnosis}

The pathological damage of the gut, demonstrated by postmortem examination of chickens was one of the diagnosis of coccidiosis. Second, diagnosis include fecal examination. ${ }^{22}$ The pathophysiological of the gut occur developmental stages of oocytes before shed the feces, some species of Eimeria does not indicate serious pathogenic condition when there was presence of large number of oocytes for instance E. tenella. E. acervulina is more oocytes than E. necatrix and high biotic potential the shed in the feces and presence of large number of oocytes. The diagnosis, isolation and identification of oocytes of Eimeria species in various species of poultry coccidia in not easy task. ${ }^{22}$ 


\section{Detection of oocyte in feces}

Identification of oocytes in the feces could be used floatation method either saturated salt or sugar solution for diagnosis of coccidiosis and is reliable method, for examination infected chicken or birds with oocytes identification in the feces. This method is useful and indicator for subclinical infection. This method performed for collection of intestinal content of chickens for screening concentration floatation techniques for further identification in Eimeria oocytes. Isolation of oocytes measurements by using a calibrated ocular micrometer at 400x magnification and location for characterization of intestinal lesion to identify different species of Eimeria, morphology, sporulation time of Eimeria species. ${ }^{23}$

\section{Examination of gross lesions}

Further diagnosis and identification of oocytes was examination gross lesions for looking characteristics of lesions, location of lesion of intestinal tract and their appearance and color and content with blood, mucosa, pus, or hemorrhage associated gross change is most reliable diagnosis of Eimeria species of coccidiosis. ${ }^{7}$

Postmortem examination procedure for killing birds. ${ }^{24}$ During performing postmortem examination of chickens required examination whole tract from gizzard to the lower rectum with strong light for clear vision of what pathological external serosal surface of the digestive tract. This examining with serosal surface could be performed found whitish plaques or petechiae. Whitish streaks or rounded colonies of oocytes in the duodenal area, this pathological change is a characteristics of E. acervulinea or E. mivati, other characteristics of E. necatrix schizonts in their mid-gut area on both sides of the yolk sac diverticulum, whitish plaques may be produced by colonies. ${ }^{25}$

Mucosa or submucosa parasitic indicating invasion of tissue become thickened for pathological effect when cutting during post mortem examination, other lesion found include mucus, blood, casts, cores and presence of cheesy of coagulation necrosis found. In the caeca diagnostic examination of E. tenella stained blood in the caeca, this blood comes from anterior tissue of the intestine and down to cecum this led to a misdiagnosis case of E. necatrix as E. tenella infections. As differential diagnosis histomoniasis, Hemorrhagic Syndrome and ulcerative and necrotic enteritis may also produce somewhat similar gross lesions. ${ }^{4}$

The gross lesions caused coccidian mostly scoring in the caeca by E. tenella can differentiated with developed post mortem examination techniques. Remove from gastrointestinal tract include gizzard and rectum and observation orientation to identify lesion location and damage various parts of the intestine the lesions are score 1 up to 4 based key identification characteristics. ${ }^{25}$

\section{Serological and molecular diagnosis}

The advanced molecular development has significance and accuracy diagnostic tool for identification of coccidiosis, polymerase chain reaction (PCR) was molecular techniques based for assay targeting different region of genome of Eimeria species. such as the $5 \mathrm{~S}$ ribosomal ribonucleic acid (rRNA) ( the small subunit rRNA the sporozoite antigen gene EASZ240/160, ${ }^{26}$ internal transcribed spencer (ITS-1 $)^{27}$ and ITS-2, ${ }^{28}$ in different genomic region of coccidiosis. Since the ITS regions are less conserved than the rRNA genes, detecting variations in this region of DNA sequence, makes the design of primers straightforward and reduces the risk of cross reactions among different species. ${ }^{29}$

Molecular method helps full demonstration of epidemiological study of parasite has been investigated for differentiation and identification of Eimeria species. complex species of Eimeria in birds can be useful to detect, targeting genome and quantify species for advanced molecular techniques of real time (PCR). Real time (PCR) has its exceptional sensitivity unlike traditional PCR assay this can be quantified. 2016 published applied real time PCR technology for infection of Eimeria species of E. maxima, and E. acervulina. ${ }^{30}$ For study both host immunity and efficacy field diagnostic for infected birds of Eimeria species.

Second molecular diagnostic test was ELISAs detect antibodies in blood (sera) samples to detect species antibodies or antibodies that recognize a range of Eimeria species. ELISAs can detect antibody not themselves species parasites. The advantage of ELISA was able to detect accurately developed an immune response when vector parasites infected with host or birds when the parasites no longer present. The efficacy vaccination and immune response can be ability to assess this efficacy provide by ELISAs, although the ELISAs high, cheap assays that would allow rapid screening in large flocks or birds. In researchers in Japan developed by E. necatrix on species-specific ELISAs kits works. ${ }^{31}$ Their test uses a recombinant antigen, so it is specific to E. necatrix.

Generally, the following characteristics are useful in Differential identification of each species of poultry Eimeria, localize intestinal parasitized, this causes damage and lesion gross appearance in the naked eye. Sporulation time, schizont size and their oocytes development and characterization oocyst morphology. Location of parasite in the host intestinal epithelium and their immunization they are illustrated in Figure 2.

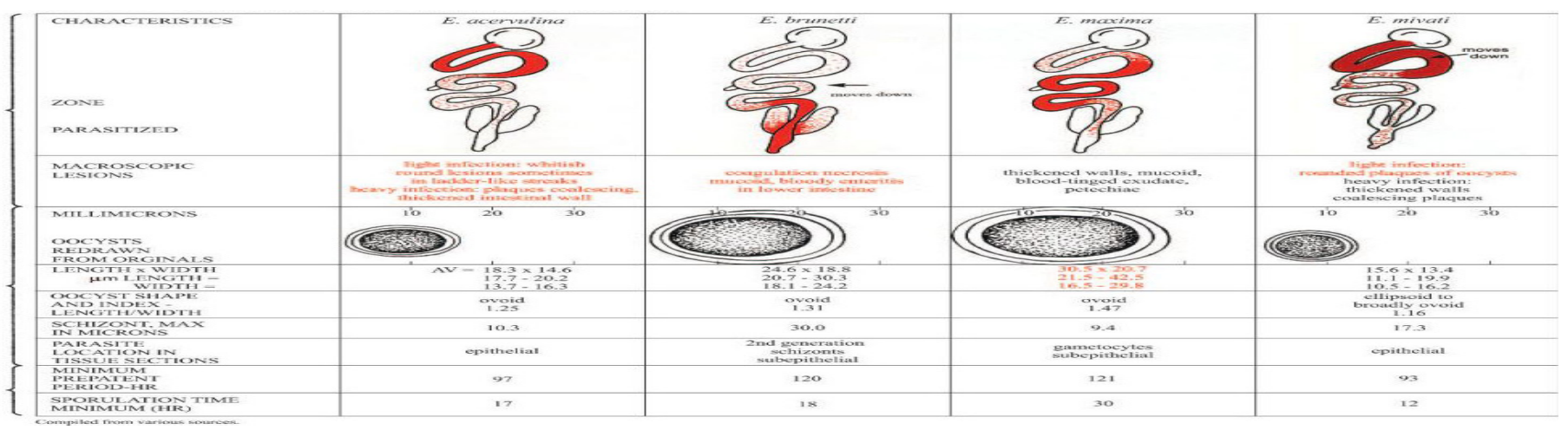




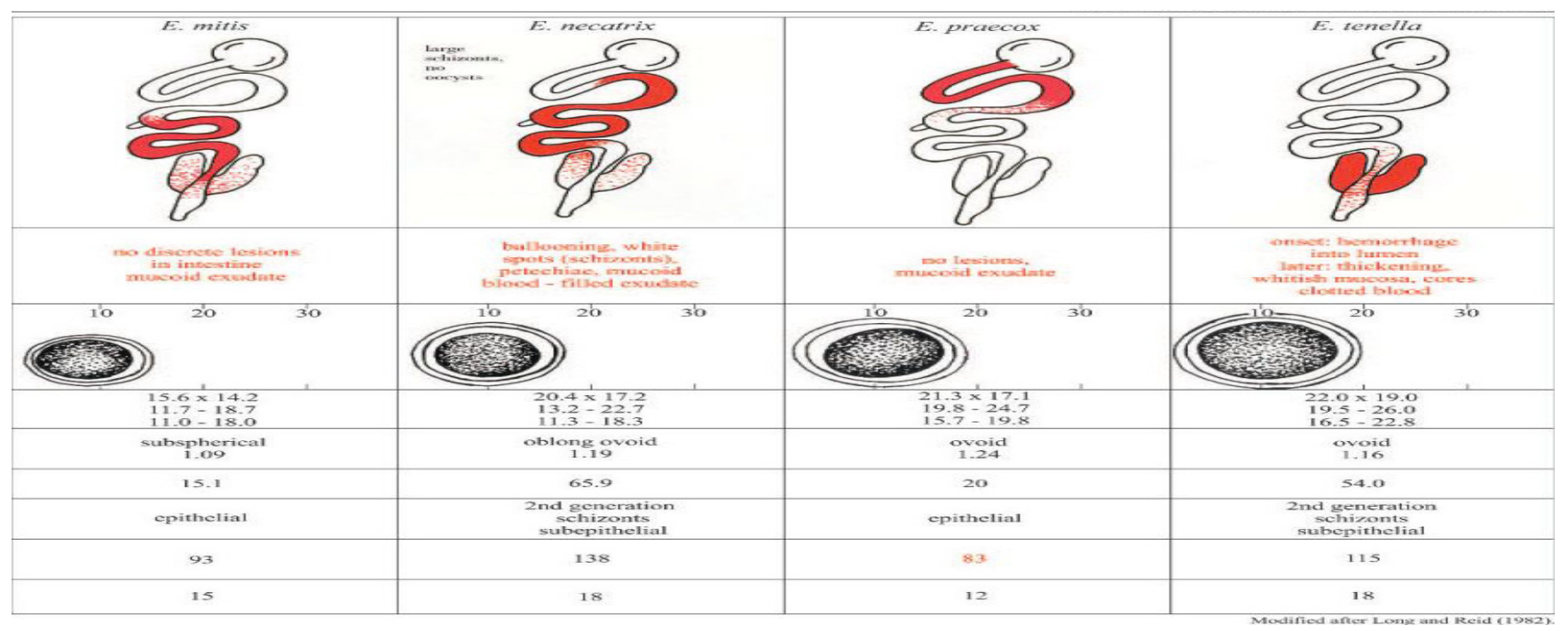

Figure 2 Differential characteristics for the 8 species of Chicken Eimeria [Source: 7].

\section{Treatment, control and prevention}

The treatment of coccidiosis is effective practice for reduce the prevalence and outbreaks of coccidiosis and have been significance role in past 50 years. The anticoccidial for feed additives have played major role in promotors growth of industry poultry and increase their quality and availability for consumers of poultry products. The chemical synthesis of anticoccidials have specific target receptors and mechanism of actions against the metabolism of the parasites (such as amprolium, clopidoldecoquinate, halofuginone, and polyether ionophores such as monensin lasalocid, salinomycin, narasin, and maduramycin, which act through general mechanisms of altering ion transport and disrupting osmotoic balance. The chemical compound has mechanism control for coccidiosis. The most frequently available and used drug was a compendium through Janssen Pharmaceutical, it is quite clear. ${ }^{32,33}$

The anticoccidial drug have degree of resistance which developed, for instance ionophores resistance has developed. In order to minimize these resistances of anticoccidials they must use and rotate various anticoccidials with successive flocks in poultry producers, the combination of chemical anticoccidials with ionophore treatment or employ shuttle programs during flock growth reduce the resistance of anticoccidials. These resistance of anticoccidials depends on seasonal conditions and prevalence of various species of the coccidia. ${ }^{32}$

\section{Control and prevention}

The most tool for control of coccidiosis could be applied with anticoccidial drug for control Eimeria species, the second, control of coccidiosis is available vaccine for breeders or layer replacement. The anticoccidial drug is very effective mechanism of control but later develop drug resistance has been affected. Frequent used of anticoccidial drugs for control of coccidiosis always relied up on to resistance of anticoccidial drugs. ${ }^{34}$

The hygiene is one control mechanism of coccidiosis apart from the use of drugs, the other methods for control of coccidiosis was vaccine and study for genetics resistance species of coccidiosis. But genetics is a theoretical strategy not in practical use. ${ }^{9}$ Immediate treatment and other methods or programs for preventive measurement is an optimal overall preventive program achieved. ${ }^{35}$ Due to the frequent chemical anticoccidials for traditional control methods are becoming less attractive could increase parasite resistance. Alternative production systems for the environment in food, and the demands control and prevention of coccidiosis. (e.g. organic). ${ }^{36}$

An alternative program which reduces resistance for chemical control by producing species-specific protection. Live vaccine is an effective method for control coccidiosis and enhance immunity of the flocks or birds include long-term, economical protection against diseases; provision of an alternative to chemical control to minimize residue and withholding period problems for meat and eggs, is effective tool to manage chemical resistance. ${ }^{36}$

Protective immunity for Eimeria parasites, with low number with infected chicken, has induced after two or three consecutive infections. ${ }^{37,38}$

\section{Conclusion and recommendation}

Eimeria is protozoan parasite infection caused by coccidiosis, cause damage tissue and intestinal tract leading dehydration and blood loss, Coccidiosis has effect on the production, productivity and economy of the world in both farmers and household levels, Coccidiosis cause immune suppression and increase other opportunistic bacterial disease. This Eimeria is caused damage intestinal epithelial tissue and caused hemorrhage, and dehydration. Diagnosis include identification oocytes in floatation and postmortem examination, examination of gross lesions, serological and molecular diagnosis. There differential diagnosis includes histomoniasis, Hemorrhagic Syndrome and ulcerative and necrotic enteritis. For the treatment of the coccidiosis is effective anticoccidial feed additives in both chemicals and ionophore though there were resistances. Apart from the drugs, other successful control and prevention of Coccidiosis include hygienic parameters, $\log$ acting immunization vaccine and study of genetics in different geographical areas. Above followed conclusion I forwarded in this recommendation: to improve molecular and serological rapid, easy diagnostic test and strategical frame work of the world for eradication of this parasitic disease.

\section{Acknowledgments}

None. 


\section{Conflicts of interest}

The authors declare there are no conflicts of interest.

\section{Funding}

None.

\section{References}

1. Julie DH. Coccidiosis in poultry. Livestock Poultry Health Programs. $1999 ; 2: 3-4$.

2. McDougald LR. Intestinal protozoa important to poultry. Poult Sci. 1998;77(8):1156-1118

3. Kaufmann J. Parasitic Infections of Domestic Animals. Germany Birkhauser. 1999:341-342.

4. Jones CT, Hunt DR, King WN. Veterinary pathology. 6th ed. USA: Lippincott Williams and Wilkings; 1996:552.

5. Conway DP, Mckenzie ME. Poultry coccidiosis, diagnostic and testing procedures. 3rd ed. Ames: Iowa black well publishing; 2007:37-40.

6. Fanatico A. Parasite management for natural and organic poultry coccidiosis. 2006.

7. Jordan F, Pattison M, Alexander D, et al. Parasitic diseases. In: Poultry Disease. 5th ed. Hong Kong: W.B. Saunders; 2002:405-420.

8. Shirley MW, Harvey DA. A genetic linkage map of the Apicomplexan protozoan parasite Eimeria tenella. Genome Res. 2000;10(10):15871593.

9. Ouarzane M, Labbe M, Pery P. Eimeria tenella: cloning and characterization of cDNA encoding a s3a ribosomal protein. Gene. 1998;225(1-2):125-130.

10. Hofstad MS. Diseases of Poultry. 8th ed. Ames, USA: Iowa State University Press; 1984:692-717.

11. Taylor MA, Coop RL, Wall R. Veterinary parasitology. Oxford UK Blackwell Publishing; 3rd ed. 2007:475-483.

12. Charlton BR. Coccidiosis. In: Avian disease manual. 5th ed. Internationa book distributing company in association with American association of avian pathologist, USA; 2006:153-156.

13. Urquhart GM, Armour J, Dunkan JL, et al. Veterinary Parasitology. UK Longman Group UK Ltd. 1987:345.

14. Jeurissen SHM, Janse EM, Vermeulen AN, et al. Eimeria tenella infections in chickens: aspects of host-parasite interaction. Vet Immunol Immunopathol. 1996;54(1-4):231-238.

15. Yun $\mathrm{CH}$, Lillehoj HS, Lillehoj EP. Intestinal immune responses to coccidiosis. Dev Comp Immunol. 2000;24(2-3):303-324.

16. Lawn AM, Rose ME. Mucosal transport of Eimaria tenella in the cecum of the chicken. J Parasitol. 1982;68(6):1117-1123.

17. Daszak P. Zoite migration during infection: parasite adaption to host defences. Parasitol Today. 1999;15(2):67-72.

18. Kahn CM. The Merck Veterinary Manual. 9th ed. White house station, NJ, USA: Merck and CO INC; 2005:2201-2206.

19. McDougald LR, Reid WM. Coccidiosis in Diseases of Poultry. 10th ed Iowa State University Press; 1997:865-883.
20. Marquardt CW, Demaree SR, Grieve BR. Parasitology and vector biology. 2nd ed. USA: San Diego, London, Boston, NewYork, Tokyo, Tornto. 2000:152.

21. Soulsby EJL. Helminths, Arthropods and Protozoan's of Domesticated Animals. 7th ed. London: Bailliere Tindall; 1982:630.

22. Zander DV, Mallinson ET. Principles of disease prevention: diagnosis and control. In: Calnek BW, editors. Disease of Poultry. 9th ed., Iowa State University Press; 1978:3-4.

23. Conway DP, Mckenzie ME. Poultry coccidiosis, diagnostic and testing procedures. 2nd ed. The Netherlands: Pfezer INC; 1991:187-200.

24. Molloy JB, Eaves FW, Jeston PJ, et al. Detection of Eimeria acervulina using the polymerase chain reaction. Avian Dis. 1998;42(1):119-123.

25. Schnitzler BE, Thebo P, Tomley F, et al. PCR identification of chicken Eimeria: a simplified readout. Avian Pathol. 1999;28(1):89-93.

26. Lien YY, Sheu, SC, Liu HJ, et al. Cloning and nucleotide sequencing of the second internal transcribed spacer of ribosomal DNA for three species of Eimeria from chickens in Taiwan. Vet J. 2007;173(1):186-191.

27. Holmdahl OJM, Mattsson JG. Rapid and sensitive identification of Neospora caninum by in vitro amplification of the internal transcribed spacer 1. Parasitology. 1996;112(2):177-182.

28. Swinkels, WJC, Post J, Cornelissen JB, et al. Immune responses in Eimeria acervulina infected one-day-old broilers compared to amount of Eimeria in the duodenum, measured by real-time PCR. Vet Parasitol. 2006;138(3-4):223-233

29. Onaga H, Kawahara F, Umeda K, et al. Field basis evaluation of Eimeria necatrix specific enzyme-linked immune sorbent assay (ELISA) for its utility in detecting antibodies elicited by vaccination in chickens. $J$ Vet Med Sci. 2005;67(9):947-949.

30. Chapman HD. Sensitivity of field isolates of eimeria to monensin following the use of a coccidiosis vaccine in broiler chickens. Poult Sci. 1994;73(3):476-478.

31. Chapman HD. Evaluation of the efficacy of anticoccidial drugs against eimeria species in the fowl. Int J Parasitol. 1998;28(7):1141-1144.

32. Vegad JL. Poultry coccidiosis. In: Poultry Diseases, a guide for farmers and poultry professionals India. International Book Distributing Company; 2004:186-197.

33. Whiteman CE, Bickford AA. Coccidiosis. In: Avian Disease Manual. 3rd ed. The American Association of Avian Pathologists 1989:385-401.

34. Chapman HD. Biochemical genetic and applied aspects of drug resistance in Eimeria parasites of the fowl. Avian pathol. 1997;26(6):221-244.

35. Joyner LP, Norton CC. The immunity arising from continuous low-level infection with Eimeria tenella. Parasitology. 1973;67(3):907-913.

36. Long PL, Johnson J, Mckenzie Me, et al. Immunization of young broiler chickens with low level infections of Eimeria tenella, E acervulina or E. maxima. Avian Pathol. 1986;15(2):271-278.

37. Anne F. Parasite management for natural and organic poultry: coccidiosis National Center for Appropriate Technology (NCAT) Agricultural Specific Journal. 2006;245:1-12.

38. Amit KP. Phytogenic approach to safeguard the birds from coccidiosis. Poultry Industry; 2018. 